\title{
DESCRIPTION OF PATIENTS' COMPLIANCE IN ASEER REGION, KINGDOM OF SAUDI ARABIA
}

\author{
Department of Clinical \\ Pharmacy, \\ College of Pharmacy, \\ King Khalid University, \\ Abha, PO. Box : 80260 \\ Jeddah. 21589 \\ Kingdom of Saudi Arabia
}

Submitted: $14-05-2015$

Revised: 25-07-2015

Accepted: 20-08-2015

*Corresponding author Khaled. M. Alakhali

Email:

khaled_akhali@yahoo.com

\author{
Khaled M. Alakhali
}

\begin{abstract}
Medication non-adherence can cause impairments that interfere with self-care activities in individuals who suffer from multiple comorbid diseases that require careful management. The objective of this study is to assess and describe medication adherence in patients of Aseer Region of Kingdom of Saudi Arabia (KSA). Samples of 416 patients in the outpatient department at Abha General Hospital and Aseer Central Hospital are selected randomly. The patients were interviewed using seven a selfadministered close-ended anonymous questionnaire. Only descriptive statistics is reported in this study for analyze the compliance category. The participants in the study were $71 \%$ (295 out of 416 patients) of participants were females, while $29 \%$ (121 out of 416) were males. The participants' age ranges between 18 60 years. The adherence level was $85 \%$. The major reasons for non-adherence were forgetfulness and feeling good. The study revealed the appropriate level of adherence by participants. The study also revealed that pharmacists and other healthcare professional intervention will help to improve further the medication adherence with possible fruitful therapeutic outcomes of the patients.
\end{abstract}

Key words: Non-adherence patient, Kingdom of Saudi Arabia

\section{INTRODUCTION}

Around a quarter of patients do not take their medicines as prescribed (Elliott et al., 2008). Non-compliance with medication means failure on the part of a client to follow the recommendations of a health professional with regards to their medication, However modern health care is concerned with working with clients and has therefore suggested that 'concordance' should replace the use of the word 'compliance'. Concordance projects patient rights, needs for information, the importance of two-way communication and decision-making such as stopping medication even if clinicians do not agree with the decision (Gray et al., 2002). The significance of the problem is indicated in statistics showing that up to half of all patients fail to follow their prescribed drug regimens (Barbara Worsley, Vice President, Risk Management, The SCPIE Companies 2014). A vast amount of research has explored its causes, and numerous factors related to the patient, disease, treatment, health care provider and health care system are known to be associated with adherence (Alakhali et al.,
2013, Dodd et al., 2012). The World Health Organization (WHO) categorized the determinants of non-adherence into five dimensions: social and economic, health system-related, therapy related, conditionrelated, and patient-related (Hovstadius et al., 2011). Research over the past 2 decades has consistently shown that intentional nonadherence is driven by patient beliefs about their treatment, disease, and prognosis as well as their objective experiences with medications. Some early research on unintentional nonadherence suggested it was more strongly correlated with demographic characteristics than with medication knowledge or beliefs (Gadkari et al., 2012). When looking specifically at the management of certain chronic medical conditions such as diabetes, hypertension and dyslipidemia, nearly one out of every three patients was primarily non-adherent (Fallis et al., 2013). Despite these insights, thus far there remains little consensus on standardized and practical approaches for assessing medication non-adherence. In part this is due to the lack of reliable, validated measurement methods that 
are useful in both research and clinical settings. Currently, the most commonly used approaches include self-report, pill counts, biological drug levels, pharmacy refill data, and electronic pill bottles, each of which has shortcomings (Ye et al., 2012). Thus improving adherence enhances patients' safety. It is crucial for health professionals both to assess the patient and foresee the possible causes of nonadherence and follow a policy for increasing medication adherence and achieving the best health outcome (Kalogianni et al., 2011). However the paucity of data about the noncompliance of patients regarding the medication use has prompted us to evaluate the reasons of non-compliance of patients in Aseer region of Kingdom of Saudi Arabia. The objective of this study is to assess and describe medication adherence in patients of Aseer Region of Kingdom of Saudi Arabia (KSA).

\section{MATERIAL AND METHODS}

The total number of patients is 416 . The study is conducted in the outpatient department at Abha General Hospital and Aseer Central Hospital, KSA, for a period of 4 months (July to October, 2014). The included patients were above 18 years and those below the age of 18 years old were excluded. Data analysis was carried out using Microsoft excel. Data were expressed as mean (standard deviation) for continuous variables and as frequency for categorical variables. Only descriptive statistics is reported (means with 95\% confidence intervals).

An anonymous hand written questionnaire was administered to 416 patients of different age groups in both Abha General Hospital and Aseer central hospital. The questionnaire comprised 7 items and the patients were required to select one or more of the reasons of non-adherence.

\section{RESULTS AND DISCUSSION}

Seventy one percent (295 out of 416 patients) of participants were females, while $29 \%$ (121 out of 416) were males. The distribution pattern of diseases among the participants (Table I). Grouping adherence levels, just 33 percent earn an A grade for being completely adherent, additional 52 percent are somewhat non-adherent (a grade of B) and the remaining 15 percent are largely non-adherent (a grade of C) (Table II). In the general population, the principal reasons for nonadherence seem to be the reasons described in Table 3. Majority of the participants is totally aware or just aware of the consequences of non-adherence described in Table 4. Adherence is defined as the extent to which a patient's behaviour or action coincides with the advice received (Wroth et al., 2006). The results of this study indicate that, as elsewhere in the world, there is a problem of non-adherence to medication among patients with in Aseer region of Saudi Arabia. Respondents' ages ranged from 18-60 years. The majority of the participants were within the age group 18-28 years. The characteristics of the diseases among the participants were as described in the Table 1. $38(9.1 \%)$ of the patients were hypertensive and diabetic, $15(3.6 \%)$ had heart diseases, 75 $(18 \%)$ pulmonary diseases, fractures and bone disease 42 (10.1\%), $109(26.2 \%)$ had internal diseases and 137 (32.9\%) had other diseases. The reasons reported by patients for nonadherence to their medication varied across the individual, but the most frequently reported reasons were forgetfulness 190 patients $(32 \%)$. This problem of forgetfulness can be resolved by using tools like medication reminder or dairy keeping. Eighty patients $(13 \%)$ of the patients reported that fear of side effects was the undue cause for non adherence. This can be solved by bringing to the doctor's attention any discomforts they are having, the doctor may change the medication or the dosage, prescribe another medication to counteract side effects, or offer practical suggestions for alleviating the discomforts. Fifty one patients $(9 \%)$ of the patients have reported the high cost of medication as the reason for non-adherence to use of medicines. These results are in agreement with the previous results (Ih Eze et al., 2011). This problem can be resolved by switching brand name medications to generic or cheaper alternatives. Reviewing the regimen for medications that may no longer be necessary can streamline the regimen for ease and cost purposes (Latif and Mcnicoll, 2009). Use of multiple medications (polypharmacy) is associated with an increased risk of complex dosing schemes (Hugtenburg et al., 2013). 
Tabel I. Patient Characteristics Table: 1 Diseases among the participants' patients in Aseer region

\begin{tabular}{clcc}
\hline SI.No & \multicolumn{1}{c}{ Disease } & Count & $\mathbf{\%}$ \\
\hline $\mathbf{1}$ & HTN \& Diabetes & 38 & $9.1 \%$ \\
$\mathbf{2}$ & Heart Disease & 15 & $3.6 \%$ \\
$\mathbf{3}$ & Pulmonary Disease & 75 & $18 \%$ \\
$\mathbf{4}$ & Fractures \& bone disease & 42 & $10.1 \%$ \\
$\mathbf{5}$ & Internal Diseases & 109 & $26.2 \%$ \\
$\mathbf{6}$ & Others & 137 & $32.9 \%$ \\
\hline
\end{tabular}

Table: 2 Patient adherences to medications in Aseer region

\begin{tabular}{cccc}
\hline Adherence & Count & $\%$ & Grade \\
\hline Always & 135 & $33 \%$ & $\mathrm{~A}$ \\
Sometimes & 217 & $52 \%$ & $\mathrm{~B}$ \\
Rarely & 64 & $15 \%$ & $\mathrm{C}$ \\
\hline
\end{tabular}

Table 3: Distribution of causes for patient non-compliance in Aseer region

\begin{tabular}{clcc}
\hline SI.No & Reason & Count & $\mathbf{\%}$ \\
\hline $\mathbf{1}$ & Difficult method of use & 23 & $4 \%$ \\
$\mathbf{2}$ & High cost and not accessible & 51 & $9 \%$ \\
$\mathbf{3}$ & A lot of medicines daily & 60 & $10 \%$ \\
$\mathbf{4}$ & Side effects or fear of side effects & 80 & $13 \%$ \\
$\mathbf{5}$ & Not feeling better with medicine & 85 & $14 \%$ \\
$\mathbf{6}$ & Feeling good, so why take medicine? & 109 & $18 \%$ \\
$\mathbf{7}$ & Underestimation of consequences, and forgetting & 190 & $32 \%$ \\
\hline
\end{tabular}

Table: 4: Awareness of consequences of non-adherence in Aseer region

\begin{tabular}{ccc}
\hline State & Count & $\%$ \\
\hline Totally Aware & 184 & $44 \%$ \\
Little Aware & 175 & $42 \%$ \\
Not Aware & 57 & $14 \%$ \\
\hline
\end{tabular}

This also has been reported in our study as one of the reasons for non-adherence by 60 patients $(10 \%)$. However patient must be educated about the need to manage potential drug-drug interactions which may also result in complex dosing schemes. Medications with a convenient way of administration (eg, oral medication) are likely to make patients compliant (Jin et al., 2008). However $23(4 \%)$ of the patients in our study complained that difficulty in using the medicine by the method described, (for example the use of inhalers in asthma cases), was also one of the reasons for non-adherence and the results are in agreement with previous studies (Al-Jahdali et al., 2013). Intentional nonadherence (not taking medications when feeling better or worse) accounted to $109(18 \%)$ and 85 $(14 \%)$ in our study.

Thirty three percent $(33 \%)$ of the patients mentioned that they are always adherent; $52 \%$ patients were sometimes adherent while taking medicines and around $15 \%$ were rarely adherent. Overall around $85 \%$ of the patients were fairly adherent while taking medicines and this result is in agreement with the previous studies (Okoro and Ngong, 2012).

Respondents attitude towards awareness of consequences of non-adherence showed that $44 \%$ of the patients were totally aware of the consequences of the missed doses, $42 \%$ were little aware and $14 \%$ were not aware of the consequences of the non-adherence to 
medication regimen. However in our study we did not record what action was taken by the patients towards the missed does being aware of the consequences of non-adherence. The positive attitude towards the missed does will enhance adherence. Therefore, noncompliance with medication regimens can result in the increased use of medical resources such as nursing homes, hospitals, physician visits, and unnecessary treatment. Noncompliance with medication regimens may also result in therapeutic failure (Alakhali et al., 2013). For example, missed doses of hypertension, diabetes and heart disease can make patients worse. Further studies for longer time period and with a larger patient number are recommended to have more definite conclusions and to determine the extent and outcome of reasons for non-compliance in patients of Aseer region and other regions in Saudia Arabia.

\section{CONCLUSION}

Non-compliance with medication is a complex and multidimensional health care problem. Patient education is the only way to improve compliance. Pharmacist's and health care professionals should enhance medication adherence and outcomes by engaging in pharmaceutical care activities such as monitoring symptoms, providing medication counseling, helping resolve drug-related problems, and facilitating communication with physicians.

\section{ACKNOWLEDGMENT}

We are thankful to the Department of Clinical Pharmacy, College of Pharmacy, King Khalid University for providing the support for this research work

\section{REFE RE NCES}

Al-Jahdali H., Ahmed A., Al-Harbi A., Khan M., Baharoon S., Bin SS., Halwani R., Al-Muhsen S., 2013. Improper inhaler technique is associated with poor asthma control and frequent emergency department visits. Allergy Asthma Clin Immunol 9, 8.

Barbara W., Vice P., Risk M., 2014. The SCPIE Companies. Noncompliance: A Problem for Patients and Doctors https://www.scpcf.com/ PDF/MLMNoncompliance.pdf (Accessed 8 March)

Dodd S., White IR., Williamson P., 2012. Nonadherence to treatment protocol in published randomized controlled trials: $a$ review. Trials 18, 13-84.

Elliott RA., Shinogle JA., Peele P., Bhosle M., Hughes DA., 2008. Understanding medication compliance and persistence from an economic perspective. Value Health 11, 600-610.

Fallis BA., Dhalla IA., Klemensberg J., Bell CM., 2013. Primary medication nonadherence after discharge from a general internal medicine service. Plos One 8, e61735.

Gadkari AS., McHorney CA., 2012 Unintentional non-adherence to chronic prescripition medications: how unintentional is it really? BMC Health Serv Res 12, 98.

Gray R., Wykes T., Gournay K., 2002. From compliance to concordance:

a review the literature on interventions to enhance compliance with antipsychotic medication. J Psychiatr Ment Health Nurs 9, 277-284.

Hovstadius B., Petersson G., 2011 Nonadherence to drug therapy and drug acquisition costs in a national population--a patient-based register study. BMC Health Serv Res 11, 326.

Hugtenburg JG, Timmers L., Elders PJ., Vervloet M., van Dijk L., 2013. Definitions, variants, and causes of nonadherence with medication: a challenge for tailored interventions. Patient Prefer Adherence 7, 675-682.

Ih Eze U., Ojieabu WA., Femi-Oyewo MN., Martins OO., 2011 Evaluation of Adherence in Elderly Diabetic Hypertensive Patients. IJPI's Journal of Hospital and Clinical Pharmacy 1, 38-46.

Jin J., Sklar GE., Min SOV., Chuen LS., 2008. Factors affecting therapeutics compliance: A review from the patients's perspective. Ther Clin Risk Manag 4, 269289.

Alakhali KM., Daniel PS., Noohu AM., Sirajudeen SA.., 2013. Patient medication adherence and physician prescribing 
among congestive heart failure patients of Yemen. Indian J. Pharm. Sci. 75 (5): 557-562.

Kalogianni A., 2011 Factors affect in patient adherence to medication regimen. Health Sci.J. 5, 157-158.

Latif S., McNicoll L., 2009. Medication and non-adherence in the older adult. Rhode Island Medical Journal 92, 418-419.

Okoro RN., Ngong CK., 2012. Assessment of patient's antihypertensive medication adherence level in non-comorbid hypertension in a tertiary hospital in Nigeria. Int J Pharm Biomed Sci 3, 47-54.

Wroth THR., Pathman DE., 2006. Primary medication adherence in a rural population: the role of the patientsphysician relationship and satisfaction with care. I Am Board Fam Med 19, 478486.

Ye S., Krupka DJ., Davidson KW., 2012. Diagnosing medication non-adherence in a patient with myocardial infarction. Front Psychol 3, 267. 